\title{
EVIDENCE FOR “UNERTAN SYNDROME” AND THE EVOLUTION OF THE HUMAN MIND
}

\author{
UNER TAN \\ Cukurova University \\ Medical School \\ Department of Physiology \\ Adana, Turkey
}

\begin{abstract}
A new family exhibiting "Unertan Sydnrome" was discovered. The pedigree analysis showed marriages between relatives. This family was similar to the first one (see Tan, 2006a), providing a firm evidence for the new syndrome. The affected children showed habitual quadrupedal walking gait, that is, they walked on wrists and feet with straight legs and arms. Their heads and bodies were mildly flexed; they exhibited mild cerebellar signs, and severe mental retardation. The pedigree demonstrated a typical autosomal-recessive inheritance. The genetic nature of this syndrome suggests a backward stage in human evolution (devolution), which would be consistent with theories of punctuated evolution. The results reflected a new theory on the evolution of human beings. That is, the evolution of humans would in fact be the evolution of the extensor motor system, responsible for upright posture, against the gravitational forces. This would be coupled with the emergence of the human mind, which can be considered a reflexion of the human motor system, in accord with the psychomotor theory (see Tan, 2005a). The most important characteristic of the newly emerged human mind was the resistance against gravitational forces. This was the resistive mind, the origins of human creativity.
\end{abstract}

Keywords bipedality, evolution, human, mental retardation, mind, quadrupedality, Unertan Syndrome

Received 29 December 2005.

Address correspondence to Prof. Dr. Uner Tan, Cukurova University, Medical Faculty, Department of Physiology, 01330, Balcali, Adana, Turkey. E-mail: unertan@cu.edu.tr 


\section{INTRODUCTION}

The author has recently discovered a new syndrome and labeled it "Unertan Syndrome," which consists of three main symptoms: quadrupedal gait, severe mental retardation, and primitive language (Tan, 2005b, c; Tan, 2006a, b). Five of 19 children from a consanguineous family were affected. The pedigree exhibited an autosomal recessive inheritance. This syndrome with mild cerebellar signs and symptoms was found to be of utmost importance in relation to human evolution, including the transition from quadrupedality to bipedality.

"Unertan Syndrome" was not previously observed or described in the scientific literature. The pedigree analysis suggested that it probably occurred only once and would never re-appear. Contrary to this expectation, the author has recently discovered another family exhibiting the typical "Unertan Syndrome," suggesting that this would not be rare. The second family with the same characteristics as the first one provided evidence for the existence of the Unertan Syndrome, which will be described in the present study.

\section{METHOD}

The present work describes a second family having children with "Unertan Syndrome." The family mostly consisted of marriages between relatives (see the pedigree in Figure 1). One man and one woman exhibited quadrupedal walking gait; one man was a quadruped during childhood, but became biped later during adulthood; one man was also quadruped during childhood, but could not walk at all during adulthood.

Upon neurological examination, they were awake, the cranial nerves were intact, muscle tonus and tendon reflexes were mildly decreased; there were no extramidal signs and symptoms. They had bilateral dysmetria and disdiadochokinesis. No muscle weakness and sensory loss were observed. Cranial magnetic resonance imaging revealed mild cerebral and cerebellar atrophy in the affected children. Otherwise, no abnormalities were seen in MRI and whole-body CT examinations.

The level of consciousness and cognitive abilities were assessed using the "Mini Mental State Examination Test," standardized for the Turkish uneducated persons. The total score is 30 points. Scores in the range of 0 to 23 indicate a disturbance of cognition with varying degrees. This test measures the capabilities in five fields: orientation (date and location), registration (immediate recall of three words), attention and calculation (count backwards 


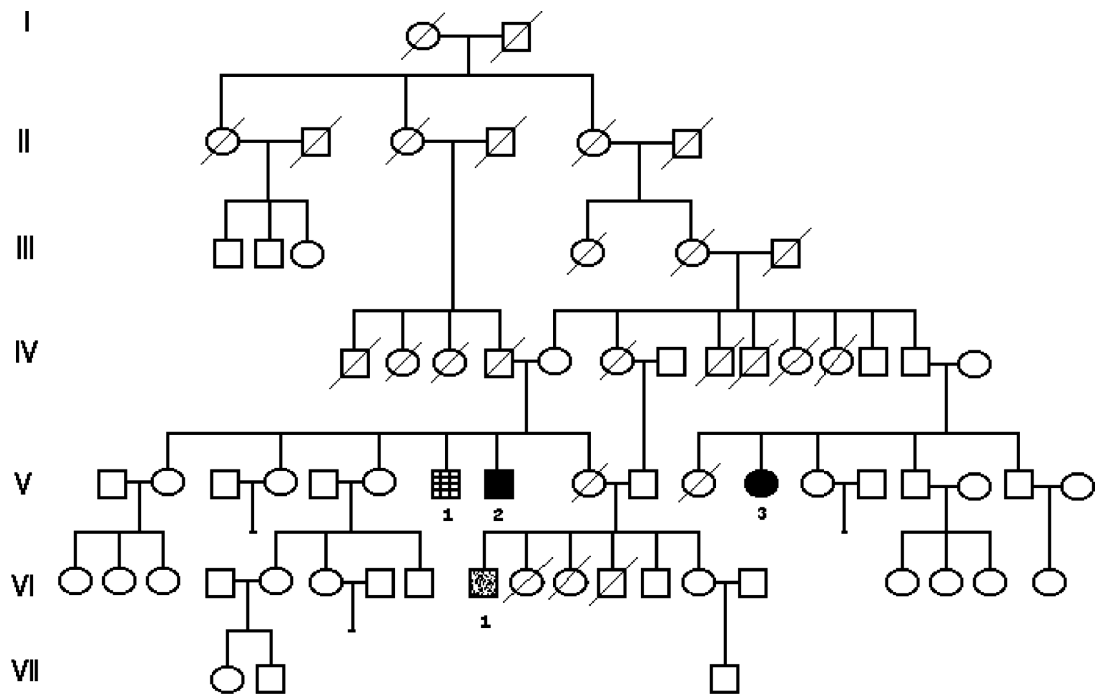

Figure 1. Family tree of the affected individuals. Open circles and open squares: unaffected women and unaffected men, being with a crossed line deceased, without a crossed line alive. Filled square: quadrupedal man $\left(\mathrm{V}_{2}\right)$; filled circle: quadrupedal woman $\left(\mathrm{V}_{3}\right)$; $\mathrm{V}_{1}$ : most severely affected man with inability to walk at all; $\mathrm{VI}_{1}$ : the man who walked quadrupedally as a child, became bipedal in his adulthood, showing ataxic gait (drunk-like), and dysmetria.

and forwards), recall three items, language, and drawing (name a few items, repeat a sentence, paper folding, draw a watch).

\section{RESULTS}

The date of examination was December 2, 2005, in the morning; the country they live in is Turkey; the region is Adana.

\section{Pedigree Analysis}

Figure 1 illustrates the family tree with affected and unaffected individuals. The affected individuals are shown with filled squares (men) and circles (women). The quadrupedal woman and man are shown by black circle $\left(\mathrm{V}_{3}, 27\right.$ years of age ) and black square $\left(V_{2}, 37\right.$ years of age $)$, respectively. $V_{1}$ is the most severely affected man (43 years of age), exhibiting total inability to walk. However, he also had a quadrupedal walking gait as a child. $\mathrm{VI}_{1}$ is the man (27 years of 
age), who had quadrupedal gait as a child, but stood up and acquired bipedal gait later in adulthood, but his gait was severely ataxic (drunk-like).

In close examination of the pedigree, one can notice that the parents of the affected children are related to each other, married with their relatives (cousins). The family tree further indicates that the Unertan Syndrome is a inherited disorder-autosomal recessive transmission. The marriages between relatives suggest that this disorder occurred in various dosages and may be a rare case.

\section{Sitting and Standing}

The top picture in Figure 2 illustrates the sitting posture of the bipedal man $\left(\mathrm{VI}_{1}\right.$ in pedigree) with ataxic gait and uncoordinated movements including dysmetria and dysdiadochokinesis. His gait was also quadrupedalal during his childhood, but later on during his adulthood he stood up and began to walk bipedally. His sitting posture was normal; his head and the upper body were not bent, exerting an upright posture. In the picture, he is attempting to take his cigarette from his pocket using the right hand. Interestingly, the contralateral hand (left hand) also moved during the movement of the right hand. This patient was usually smiling, and did not exhibit signs and symptoms of depression.

The bottom picture in Figure 2 shows the sitting posture of the quadrupedal man $\left(\mathrm{V}_{2}\right.$ in the pedigree). As clearly seen in this picture, the sitting posture was dominated by the flexor motor system, the head, arms, and hands being flexed (bent). He never stood up after his babyhood. Notice the depressive mood accompanying the flexor posture.

Figure 3 illustrates the standing postures of the quadrupedal man (left) and the bipedal man (right). The quadrupedal man could stand only using a wall; he could actually not reach a fully erect posture. His body, knees, arms, and wrists are bent, exhibiting a flexor dominance in the postural muscles. His legs were spread during standing, similar to the bipedal patient who did not need support to stand up, while he had to use spread legs to have equilibrium during standing.

\section{Walking}

The difference between the walking gaits of most primates (right) and nonprimate mammals (left) is illustrated in Figure 4. Nonprimates generally use lateral sequence (parallel) walking gaits. In contrast, most primates use diagonal walking gait. That is, the feet in the diagonal ends of the body strike 

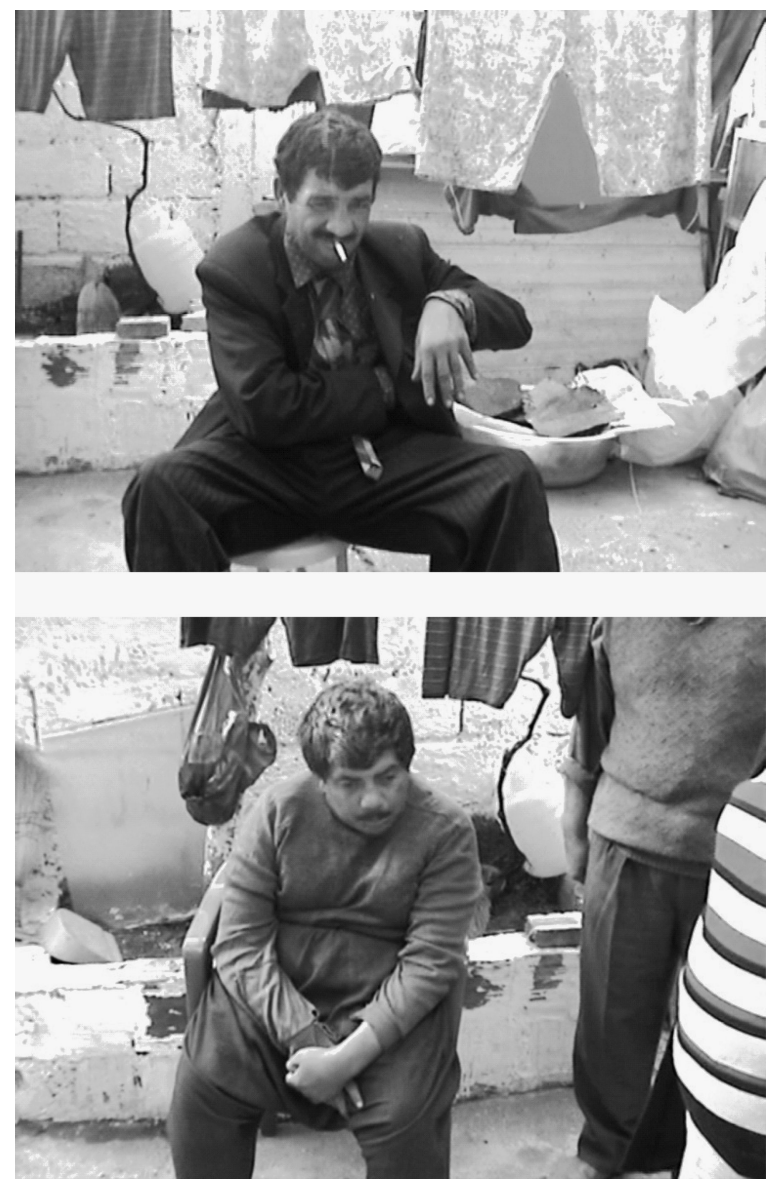

Figure 2. Typical sitting posture in the man who was originally quadrupedal in his childhood, being bipedal during his adulthood ( $\mathrm{VI}_{1}$, above), and in the quadrupedal man (bottom).

the ground together. The balance and support are maintained, for instance, by the left foot-right hand while the left hand-right leg are suspended, and then the opposite diagonal starts for further walking.

The walking gaits of the quadrupedal man and woman are illustrated on the left and right sides of Figure 5, respectively. The man exhibited a quadrupedal walking gait typical for most primates (cf. Figure 4). The arms and legs were not bent during quadrupedal walking. However, the legs were spread, probably to maintain the body equilibrium. The woman exhibited a similar walking pattern. That is, she used a diagonal walking gait, using left 

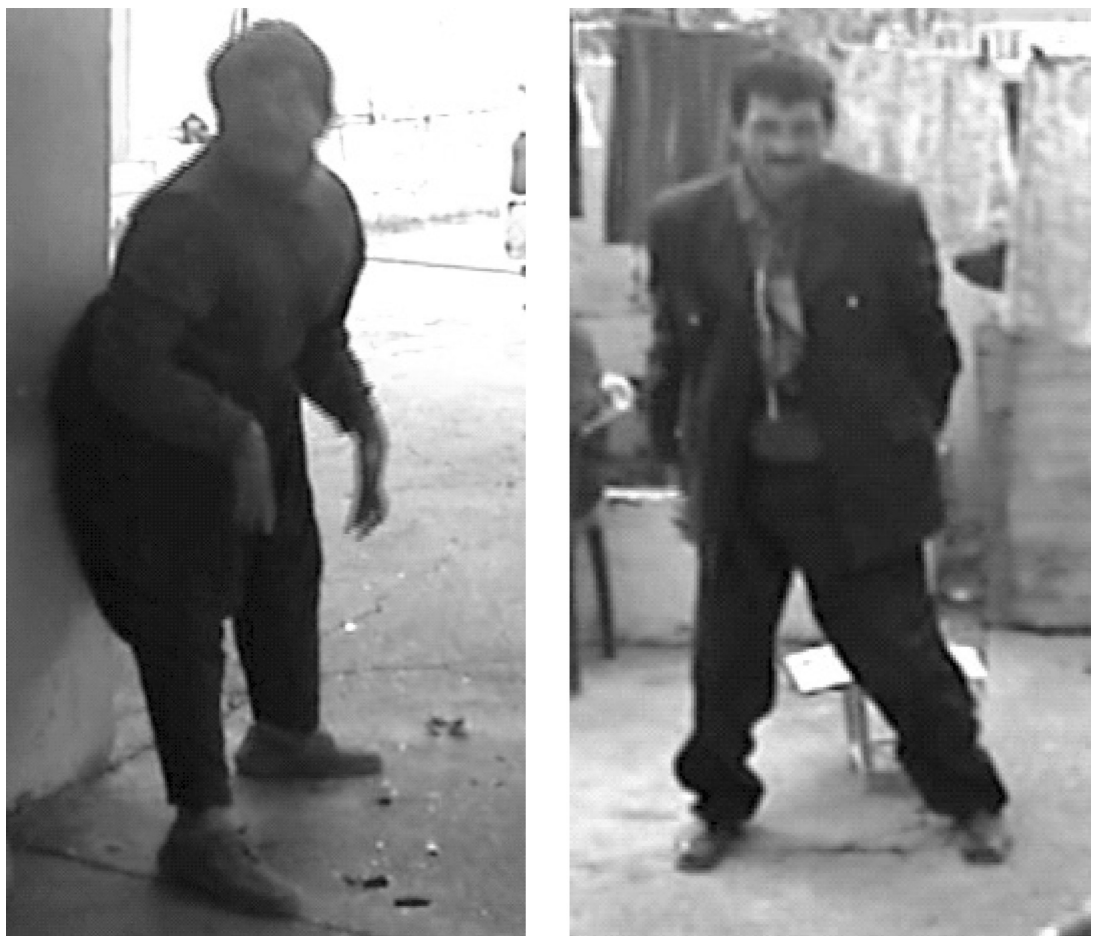

Figure 3. Standing postures in the quadrupedal (left) and bipedal-ataxic man (right).
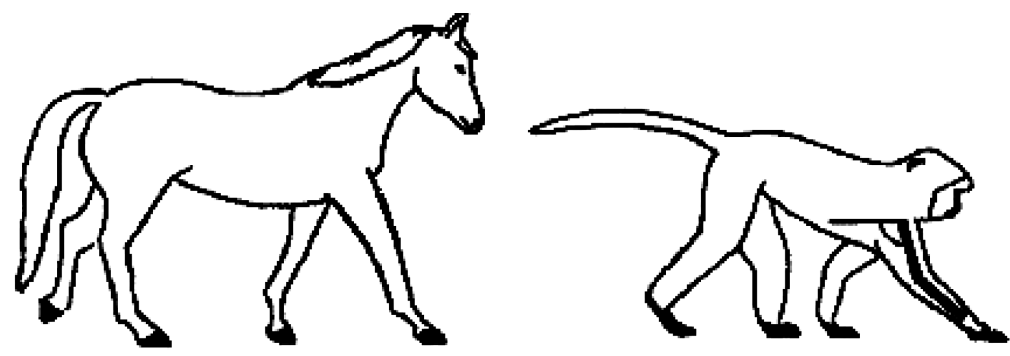

Figure 4. Differences between walking gaits of most primate (right) and most nonprimate mammals (left). Most nonprimates generally use lateral sequence walking gaits (left hindlimb-left forelimb, right hindlimb-right forelimb); most primates generally use diagonal walking gaits (left hindlimb-right forelimb, right hindlimb-left forelimb). 

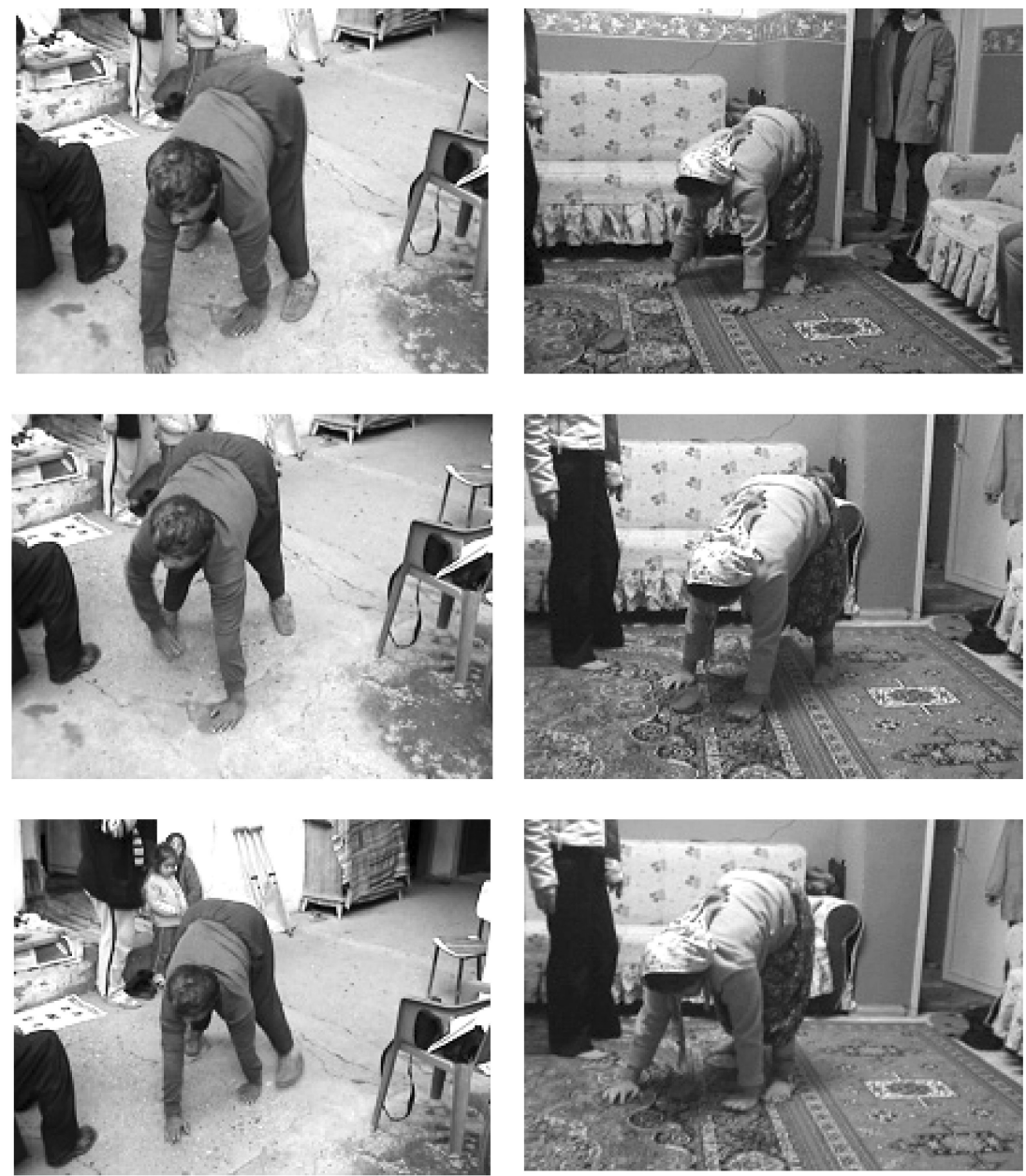

Figure 5. Habitual walking patterns in quadrupedal male (left) and female (right) patients.

hand-right foot, right hand-left foot. Whereas the man used his palms during quadrupedal walking, the woman used her fists to touch the ground. So, both quadrupedal man and woman habitually used a diagonal walking gait, which was similar to the diagonal walking pattern seen in most primates. That is, the feet at diagonal ends of the body strike the ground together, the balance and support are maintained, for instance, by the left foot-right hand while the left hand-right foot are suspended, and then the opposite diagonal starts for further walking action. Similar to most primates, they habitually used diagonal 
sequence of footfall patterns, in which the footfall of a foot is followed by that of a contralateral hand. The video clips 1 and 2 show the quadrupedal walking in man and woman, including the ataxic gait and dysmetric hand movements in the biped man with severe ataxic signs including a drunk-like gait.

\section{Speech}

Speech had an ataxic quality in all three affected individuals. There was an incoordination of the upper lips, in addition to the prolonged separation of syllables during speech. They could speak only with great difficulty in articulation. The facial muscles moved together with upper-lip muscles with an impression of laughing. They exhibited a severe dysarthria.

\section{Cognition}

The questions and the answers of the patients during the "Mini Mental State Examination Test" are presented in Table 1. The patients with quadrupedal and bipedal walking gaits had zero, and the woman with quadrupedal gait had 3 points in this test.

Table 1. Questions and answers of the patients in the Mini Mental State Examination

\begin{tabular}{llll}
\hline & & \multicolumn{2}{l}{ Patient's answers } \\
\cline { 2 - 4 } Questions & Quadr. Man & Quadr. Woman & Biped Man \\
\hline What is the year? (2005) & Today & 1,000 & 2,000 \\
What is the season? (winter) & No reply & I am & No reply \\
What is today? (Monday) & No reply & Tomorrow & Saturday \\
Morning, noon, afternoon (morning) & Evening & Evening & No reply \\
Repeat: apple, table, money & No reply & Apple & No reply \\
Which country are we living in? & No reply & USA & Germany \\
Which city are we living in? & No reply & No reply & No reply \\
Count backwards from 10 to zero & Ten & No reply & No reply \\
Count forwards from zero to 10 & Zero & Zero & Zero \\
Recall the names: tish, flag, clothe & No reply & Tish & No reply \\
What is this? (Black shoe) & Jacket & Jar & Box \\
What is this? (watch) & Leg & No reply & Me \\
What is this? (a book) & No reply & Don't know & Pencil \\
Repeat the phrase: of ifs, ands, buts & No reply & No reply & No reply \\
Take the paper, fold it in half once & & They only played around with the paper \\
\hline
\end{tabular}




\section{DISCUSSION}

The results of the present study provided evidence for "Unertan Syndrome." Namely, the author has discovered a second family with the same syndrome as the previous one (see Tan, 2005b, c; Tan, 2006a, b). The main symptoms of the Unertan Syndrome were the habitual quadrupedality (palm or wrist walking gait), severe mental retardation, and speech disturbance. There were two quadrupedally walking children in the new family. They had severe mental retardation and speech disturbance at the same time. Similar to the first family, first described by Tan (2005b, c; 2006a, b), the second family also showed that the syndrome has genetic origins exhibiting autosomal recessive inheritance.

The present study provided evidence for the existence of the Unertan Syndrome. As in the previous family, the MRI examination revealed a cerebellar atrophy with a mild cerebral atrophy. This syndrome may not be solely explained by a cerebellar atrophy because the man with a similar cerebellar atrophy did not have a quadrupedal walking gait; rather, he had a habitual bipedal walking gait despite a severe incoordination in legs and hand, such as the drunk-like gait, dysmetria, and dysdiadochokinesis.

The patients including the man with bipedal walking gait had severe mental retardation and speech disturbance. The Mini Mental State Examination Test revealed that they were disoriented in time and place and nil in arithmetic calculations and memory. The results suggest that the brain regions other than cerebellum may be involved in this syndrome. Their speech was very slow and could be understood only with great difficulty, similar to the cerebellar speech disorders.

The most pronounced postural trait was the flexor dominance during sitting and standing. As for the first family with quadrupedal walking-gait patterns, these patients may also be extremely important, as a live model, for understanding the transition from quadrupedality to bipedality in human evolution. Accordingly, the patients had apelike posture with head thrust forward, bent knees, and flexed bodies in both families. The genetic origins of Unertan Syndrome with the main symptoms of the apelike posture, quadrupedality, mental retardation, and primitive speech suggest a genetic devolution in human beings. This is consistent with the theories of punctuated evolution because the pedigree showed that a genetic mutation caused a devolution from bipedality to quadrupedality. Consequently, if one can find a gene responsible for this devolution, then one can possibly find the gene responsible for the transition from quadrupedality to bipedality in the human evolutionary history. This would be further evidence for punctuated evolution. 
In this context, some hotspots were found in human genome for acquiring duplicated DNA sequences, but only at specific time-points during evolution. It actually seems that long periods of genomic stasis are punctuated by relatively short episodes of duplicative activity; new DNA repeats arise in the genomes of species in irreversible and punctuated events (Minghetti \& Dugaiczyk, 1993).

The debate on long-term rates of evolution is accumulated around two models: the punctuated equilibrium hypothesis and the gradual thesis, the latter being referred to a gradual rate of evolution, and the former to long periods of very little evolution interrupted by periods of relatively rapid evolutionary changes. During evolution, beneficial mutations can sweep successively through the population; the most elementary processes giving rise to punctuated evolution (Elena et al., 1996). In this context, Mezhzherin (1997) has analyzed the genetic differentiation of taxa from three Holarctic and three originally Afrotropical phyla of small mammals and found that (i) distributions of fixed gene differences were relatively independent and (ii) the speciation process was discontinuous. These results were consistent with punctuated equilibria, suggesting a discontinuity in speciation process. Regarding the co-evolution of cognitive and motor systems, Wallace (2002) has reported that the learning plateaus in cognitive systems and punctuated equilibria in evolutionary process are formally analogous. These and many other similar studies are consistent with a punctuated genetic mutation, suggesting that the punctuated equilibria may play a role in the evolution of human beings.

The flexor-dominated posture in the patients with "Unertan Syndrome" exhibiting a human devolution may be specifically interesting for the evolutionary processes with regard to motor and cognitive functions in human beings. Apparently, the flexor motor system dominated the extensor motor system in patients with Unertan Syndrome, contrary to normal men with a dominant extensor motor system during sitting, standing, and walking. Therefore, human evolution would in fact be the evolution of the extensor motor system. Using this new theory, one can better understand the evolution of the human mind because the motor system is the main nucleus for the emergence of the human mind, following the psychomotor theory (see Tan, 2005a).

With regard to the evolution of the human mind, one can distinguish two important events in the human history, since splitting from our common ancestor-probably 6 to 8 million years ago. The first event was the transition from quadrupedality to bipedality with the appearance of Homo erectus with the habitual bipedal walking gait, approximately 1.5 million and 400,000 years ago. The second event was the emergence of language approximately 40,000 years ago. 
The transformation from habitual quadrupedality to habitual bipedality is the most important stage in human evolution. The habitual bipedal walking gait may be considered the most important first step predating the other uniquely human traits. The individuals with Unertan Syndrome had primitive forms of all the uniquely human characteristics, such as intelligence, articulated speech with syntax, and conscious experience. Therefore, they can provide some clues about the evolution of the human mind. The genetic nature of this syndrome, that is, a genetic mutation influencing the brain, is consistent with theories of punctuated evolution. It is reasonable to assume that there may be a gene for bipedality or a gene pool for additional human characteristics, such as articulated speech with syntax, high intelligence, and conscious experience. So, a genetic factor may be responsible for the appearance of human beings having a mind, which resisted gravitational forces. This was the resistive human mind.

The system responsible for the upright posture is the extensor motor system, consisting of the skeletal muscles holding man upright against gravitational forces. Homo erectus had the first clues of human mind co-evolved with the extensor motor system, equipped with free will to resist, first of all, the gravitation. So, the most disadvantageous factor for further development, the earth's gravitational forces, existed using the increased extensor motor system activity with the newly developed human mind.

Within the second stage of human evolution, as recently as 40,000 years ago, the appearance of human language, that is, the evolution of syntax, is seen, probably due to a mutation affecting the human brain. According to psychomotor theory (see Tan, 2005a), the human mind is an emergent property of the motor system expressed by human language. Therefore, it can be argued that the most important jump in human mind occurred with the appearance of the first modern human language, as a by-product. The enormous accomplishments of this new, suddenly emerged human mind are well known.

\section{CONCLUSIONS}

The present work has described the second family exhibiting "Unertan Syndrome" and marriages between relatives. This provided further evidence for the newly established syndrome with the main symptoms of quadrupedal walking gait, severe mental retardation, and speech disturbance. The autosomal recessive transmission of this syndrome indicates a genetic mutation causing devolution in human characteristics. This was thought to be consistent with the theories of punctuated evolution. The flexor-dominant posture of the patients, 
as the result of a genetic mutation suggests the importance of the extensor motor system in human evolution. In accord with the psychomotor theory, it was argued that a punctuated evolution in the activity of the extensor motor system may be closely coupled with co-evolution of the human mind with resistive characteristics, first of all against the earth's gravitational forces. Homo erectus would be created by this newly emerged human mind. The second stage in human evolution may have been started with the appearance of human language, as recently as 40,000 years ago, due to a mutation affecting the brain. The evolution of the motor system inducing human language may be the origin of the modern human mind.

\section{REFERENCES}

Elena, S. F., Cooper, V. S., \& Lenski, R. E. (1996). Punctuated evolution caused by selection of rare beneficial mutations. Science, 272, 1802-1804.

Mezhzherin, S. V. (1997). Gradualism or punctualism: Data on genetic differentiation of small mammals from the Holaretic region. Genetika, 33, 518-523.

Minghetti, P. P., \& Dugaiczyk, A. (1993). The emergence of new DNA repeats and the divergence of primates. Proc. Natl. Acad. Sci. U.S.A., 90, 1872-1876.

Tan, U. (2005a). Psychomotor theory: Mind-brain-body triad in health and disease. In S. N. Sarbadhikari (Ed.), Depression and dementia: Progress in brain research, clinical Applications.: Hauppauge. New York: Nova Science Publishers, Inc. (pp. 21-53).

Tan, U. (2005b). A new theory of the evolution of human mind; Unertan Syndrome: Quadrupedality, primitive language, and severe mental retardation. NeuroQuantology, 4, 250-255.

Tan, U. (2005c). Unertan Sendromu ve insan ruhunun evrimine iliskin yeni bir teori. Biyobank, e. B. D. 2. 1-2.

Tan, U. (2006a). A new syndrome with qaudrupedal gait, primitive speech, and severe mental retardation as a live model for human evolution. International Journal of Neuroscience, 116, 361-370.

Tan, U. (2006b). UNERTAN syndrome (quadrupedality, primitive language, and severe mental retardation); A new theory of the evolution of human mind. International Journal of Neuroscience, in press.

Wallace, R. (2002). Adaptation, punctuation and information: A rate-distortion approach to non-cognitive 'learning plateaus' in evolutionary process. Acta Biotheor., 50, 101-116. 